\title{
The new Andean Regional Office of Astronomy for Development
}

\author{
Farid Char $^{1}$ and Jaime Forero-Romero ${ }^{2}$ \\ ${ }^{1}$ Unidad de Astronomía, Facultad de Cs. Básicas, Universidad de Antofagasta \\ Avenida U. de Antofagasta 02800, Antofagasta 1270300, Chile \\ tel.: +5655 2637596, email: farid.char@uantof.cl \\ ${ }^{2}$ Departamento de Física, Universidad de los Andes \\ Carrera 1 18-10, Bloque Ip., Bogotá, Colombia \\ tel.: +571 3394949-5183, email: je.forero@uniandes.edu.co
}

\begin{abstract}
The Andean Regional Office of Astronomy for Development (ROAD) is a new effort in South America to serve several goals in astronomical development. The six countries in the Andean ROAD (Bolivia, Colombia, Chile, Ecuador, Peru and Venezuela) represent a common language block in the region. They work together to develop strategies to strengthen the professional research, education and popularization of astronomy. Our current Working Structure comprises a ROAD Coordinator and one Coordinators in each Task Force. Here we describe the main points of the ROAD's current action plan.
\end{abstract}

Keywords. andean, office, development

\section{Introduction}

The countries in the Andean Region (Bolivia, Colombia, Chile, Ecuador, Peru and Venezuela) represent a common language block in South America, sharing a common vision about the importance of cultural development that can be achieved through regional cooperation and institutional commitment. The Andean Regional Office of Astronomy for Development (ROAD) serves this goal - strengthening ongoing collaboration efforts, creating channels of communication and developing new strategies to exchange knowledge and human resources. The participating institutions of the Andean ROAD will share research, educational and outreach visions and activities. We expect to increase the engagement with astronomy in all the Andean ROAD countries, leveraging the special role of Chile with its great conditions for astronomy and the involvement of many professional observatories, universities and astronomical institutions.

\section{Overview of Task Forces}

The Andean ROAD is under supervision of the Office of Astronomy for Development (OAD) of the IAU. The main goal of the Andean ROAD is to guarantee and strengthen effective methods of communication between the representatives and coordinators of global, regional and local projects implemented in the Andean countries, through the three Task Forces established by the OAD : (i) Astronomy for Universities and Research, (ii) Astronomy for Children and Schools and (iii) Astronomy for the Public. The ROAD Main Coordinator is Jaime Forero-Romero, $\mathrm{PhD}$, from the Universidad de los Andes (Bogotá, Colombia). The Andean ROAD's plans include several projects and ideas for each Task Forces, under supervision of a single representative, as follows:

Task Force 1. Coordinator: Germán Chaparro, PhD (Universidad ECCI, Bogotá, Colombia). 
- Andean School on Astronomy/Astrophysics. The main objective is to organize a school for advanced undergraduate and graduate students. The first version of this school was held in Quito (Ecuador) during December 2014 with great success, and we expect to develop a new version during 2016, to be held in Lima (Perú), as a joint effort between Perú and Chile.

- Andean Graduate Program. The main objective is to determine the feasibility of creating and funding an Andean graduate program. This could be a great opportunity to provide powerful experiences in astronomy for those countries without formal programs.

- Massive Open Online Courses. The main objective is to create a MOOC at the introductory undergraduate level and general public. This MOOC could also have a version for the general public, and can be considered as a TF3 project as well.

Task Force 2. Coordinator: Ángela Pérez (Parque Explora, Medellín, Colombia).

- Virtual Training Sessions. The main objective is to create virtual training and activities open to teachers and students, by developing a virtual platform to hold virtual workshops on information technologies applied to astronomy and space sciences.

- Special teachings. Design teaching material to work with visually impaired students. The first step will be a comprehensive research on this kind of material and current experiences. Later on will be defined the best way to produce and distribute these materials.

- Annual TF2 meeting. An annual meeting to gather all the collaborators in the Task Force, looking for new collaborations and alliances. The venue will change every year, with a different key topic.

Task Force 3. Coordinator: Farid Char (Sociedad Chilena de Astronomía, Chile).

- Development for planetariums. The main objective is to develop special shows for planetariums in the region, with the aim of highlighting ancestral traditions and the particular cosmovision from many communities in South America.

- Communicating Astronomy with the Public. The main objective is to organize a periodic meeting to gather all the collaborators in the Task Force. This should be a very powerful platform for all people involved in communicating astronomy to the public. The first meeting will take place at the same time as the international CAP meeting to be held in Medellín (16-20 May 2016), only a few months before the XV Latin American Regional IAU Meeting, in Cartagena de Indias (3-7 October 2016).

\section{The role of Chile and future plans}

Due to the large size of the Chilean professional astronomical community and its special role in global astronomy, the interaction to coordinate activities in Chile will be done through the Sociedad Chilena de Astronomía (SOCHIAS). SOCHIAS has designated specific representatives per Task Force, and a main coordinator of the Chilean branch. The physical address of the Chilean branch (formally Oficina Nacional de Coordinación) is located in the Unidad de Astronomía of the Universidad de Antofagasta.

We expect that the Andean ROAD will improve the overall state of astronomy development in our region following the vision of the IAU Strategic Plan 2010-2020 International Astronomical Union (IAU, 2012).

More information about our future plans, can be accessed through the official website of the Andean ROAD http://andean-road.uniandes.edu.co.

\section{References}

International Astronomical Union 2012, Astronomy for Development Strategic Plan 2010-2020. 